\title{
Um Modelo para Geração de Perfis de Usuários Baseado em Técnicas de Psi- cometria
}

\author{
Marcelo Henrique Euzebio Batista \\ Universidade do Vale do Rio dos Sinos - Unisinos Pro- \\ grama Interdisciplinar de Pós-Graduação em Computação \\ Aplicada São Leopoldo - Rio Grande do Sul - Brasil \\ marcelo.batista@sinprors.org.br \\ Jorge Luis Victória Barbosa \\ Universidade do Vale do Rio dos Sinos - Unisinos Pro- \\ grama Interdisciplinar de Pós-Graduação em Computação \\ Aplicada São Leopoldo - Rio Grande do Sul - Brasil \\ ibarbosa@unisinos.br
}

\author{
João Ricardo Bittencourt \\ Universidade do Vale do Rio dos Sinos - Unisinos Pro- \\ grama Interdisciplinar de Pós-Graduação em Computação \\ Aplicada São Leopoldo - Rio Grande do Sul - Brasil \\ joaorb@unisinos.br \\ Sergio Crespo C S Pinto \\ Universidade do Vale do Rio dos Sinos - Unisinos Pro- \\ grama Interdisciplinar de Pós-Graduação em Computação \\ Aplicada São Leopoldo - Rio Grande do Sul - Brasil \\ crespo@unisinos.br
}

\begin{abstract}
Resumo Este trabalho propõe um modelo para geração de perfis de usuários baseado em Psicometria, propiciando a junção entre áreas distintas, como Psicologia e Computação. O PPG (Psychometric Profile Generator), assim denominado, consiste em um modelo computacional para geração de perfis de usuários, prospectando o nível de habilidade ou comportamento dos avaliados através do modelo matemático TRI - Teoria de Resposta ao Item. O PPG fornece aos sistemas externos de educação, entretenimento e recrutamento e seleção o nível de habilidade ou comportamento não tendo nenhum contato com os usuários desses sistemas. Sendo assim o principal foco do PPG é fornecer um perfil na forma de um nivel de habilidade e ou comportamento, valendo-se de conhecimentos de áreas distintas, como os modelos matemáticos da Psicometria e técnicas de Inferências Estatisticas.
\end{abstract}

Palavras-Chave: Psicometria. Inferência Estatística. Educação. Entretenimento. Recrutamento e Seleção.

\begin{abstract}
This paper proposes a model for generation of profiles of users based on Psychometrics, providing the junction between different areas, such as psychology and computing. The PPG (Psychometric Profile Generator), so called, consists of a computational model for generation of profiles of users, prospective the level of skill or assessed by the behavior of model mathematical IRT - Item Response of Theory. The PPG provides external systems of education, entertainment and recruitment and selection of the level skill or behavior are not having any contact with users of such systems. Thus the main PPG's focus is to provide a profile as a level of or ability and behavior, are worth knowledge in different fields, such as models Psychometrics and the mathematical techniques of inferences Statistics.
\end{abstract}

Keywords: Psychometrics. Statistics Inference. Education. Entertainment. Recruitment and Selection. 


\section{Introdução}

Nas últimas décadas ocorreram importantes evoluções científicas, quando áreas específicas do conhecimento evoluíram consideravelmente com contribuições para o aumento médio da expectativa de vida, com avanços nos meios de comunicação, automatização e informatização de diversos setores da economia mundial.

No entanto, observa-se uma desconexão entre as ciências, nas quais as diversas áreas do conhecimento procuraram soluções isoladas em seus nichos de evolução e abrangência, deixando, assim, de contribuir ou receber contribuições de áreas distintas, como, por exemplo, a Psicologia, a Matemática, a Biologia, entre outras tantas. Essa idéia de segmentação, na qual se produz uma parte do produto final, não combina com as necessidades gerais de um ser humano, pois precisamos enxergar os contextos e suas relações.

As produções industriais, comerciais, acadêmicas e de serviços estão baseadas em fornecer ou agregar valores, seja através de um produto, uma descoberta científica, ou um serviço prestado. Nesse sentido, é necessário fornecer mais informações a clientes, pacientes, alunos e usuários, ou seja, os seres humanos que consomem os produtos e serviços possuem diferentes características, informações, preferências, idéias e sentimentos, que estão em constante mudança.

Assim, o principal elo na criação de sistemas computacionais, sejam eles educacionais, comerciais ou de entretenimento, é oferecer atratividade e equilíbrio entre as diferentes personalidades ao interagirem com esses sistemas. Para viabilizar a criação desses sistemas, surge a necessidade da construção de um mecanismo que auxilie a inferir, estimar e prospectar de maneira científica as preferências e tendências das pessoas.

Dessa forma, a união de áreas do conhecimento, no sentido de aproximar essas áreas, tais como Psicometria, Computação e Inferência Estatística, aponta para uma junção de descobertas e evoluções que se complementam, pois a Psicometria necessita de algoritmos e do processamento das informações proporcionado pela Computação, assim como se utiliza da Inferência Estatística para prospectar e comprovar a veracidade dos dados.

Este artigo apresenta um gerador de perfis de usuários para sistemas computacionais, baseado na Psicometria (PPG - Psychometric Profile Generator). O PPG usa as inferências e prospecções viabilizadas pelo modelo matemático da Psicometria, conhecido como TRI (Teoria da Resposta ao Item) para gerar o perfil para os sistemas externos ao PPG, como: provas online, sistemas de educação à distância, testes psicológicos ou jogos on-line. Esses sistemas ao receberem o perfil prospectado pelo gerador podem fornecer maiores atratividades e interatividade em seus processos educacionais, de entretenimentos e seleção de recursos humanos.

O PPG pode ser utilizado nas áreas de educação, entretenimento, comércio, recrutamento, seleção e psicologia, agregando valores, tais como: adaptação dos sistemas às preferências e evoluções dos usuários nas áreas mencionadas, conforme sua interação com os sistemas.A motivação central desse trabalho é a criação de perfis de usuários, com o foco em suas habilidades e comportamentos, procurando interação com sistemas externos ao PPG, facilitando, assim, a manipulação dos especialistas nos modelos matemáticos viabilizados pela Psicometria.

Os trabalhos realizados por psicólogos, utilizando a Psicometria como ciência que procura medir os comportamentos e habilidades definidos como traço latente, centralizam-se em obter informações de seus pacientes (clientes), através de testes e, a posteriori, de análise através de modelos matemáticos e Inferência Estatística [1].

Para viabilizar sua atuação, o PPG fornece informações aos sistemas educacionais, de entretenimento, de recrutamento e de seleção. Assim, os usuários dos sistemas não se comunicam com o PPG, pois as coletas de informações das interações dos usuários e dos sistemas ficam por conta dos sistemas externos ao PPG. Dessa forma, o PPG consegue atender a diferentes tipos de sistemas e realiza sua tarefa de gerar perfis de usuários.

Os sistemas, por sua vez, devem interagir com os usuários e o gerador. Essa interação deve ser sincronizada, uma vez que cada sistema sabe os momentos certos de coletar os itens e/ou estímulos e enviá-los ao gerador. A função do gerador é definir o nível de habilidade ou comportamento solicitado pelo sistema e armazenar essas informações para futuras interações.

O artigo está divido em sete seções, que são: a seção 2 apresenta a psicometria e o modelo matemático utilizado; a seção 3 descreve o modelo computacional e seu funcionamento; a seção 4 apresenta um estudo de caso na utilização do PPG aplicado em jogos educacionais; a seção 5 aborda os resultados obtidos com o 
estudo de caso; a seção 6 discute os trabalhos relacionados e a seção 7 as considerações finais.

\section{Psicometria}

A Psicometria é uma subárea da Psicologia que utiliza a Estatística para estudar fenômenos psicológicos. Assim o ciclo inicia com a definição pelo especialista (Psicólogo e ou Pedagogo) de qual comportamento ou habilidade será medida, auxiliando no passo seguinte, que é o de construir itens e/ou estímulos que realmente representem o comportamento ou habilidade desejada e definida no passo anterior [8].

Nesse sentido, é necessário estabelecer a dimensionalidade do comportamento ou habilidade, definir constitutiva e operacionalmente o comportamento ou habilidade em questão, operacionalizando-o em tarefas, itens de habilidades e/ou estímulos comportamentais [1].

Através do modelo matemático conhecido como TRI e representado na equação 1, é possível estimar um perfil de cada sujeito e de coletar informações sobre cada item de um teste, verificando o traço latente $\operatorname{Pi}(\theta)$ e adequando os itens para cada sujeito conforme os objetivos propostos [9].

$$
P_{i}(\theta)=c_{i}+\left(1-c_{i}\right) \frac{e^{D a_{i}\left(\theta-b_{i}\right)}}{1+e^{D a_{i}\left(\theta-b_{i}\right)}} \quad(i=1,2, \ldots \ldots, n)
$$

Na equação 1 o $\operatorname{Pi}(\theta)$ é a probabilidade de um examinando responder corretamente determinado item ou estímulo. As constantes $a i, b i$ e $c i$ são valores já prospectados pela TRI. Por sua vez, o $n$ é o número de itens aplicados aos avaliados e a constante $e$ é o número de Euler $(2,7182818)$. Finalmente, a constante $D$ possui valor de 1,7 [7].

O modelo da TRI é representado graficamente pela função na forma de uma curva característica relacionada ao item, conforme ilustrado na figura 1.

A estimativa dos parâmetros é feita usando procedimentos por meio de métodos de Máximas Verossimilhanças. As equações de estimativa, por esses métodos, não podem ser solucionadas analiticamente, por isso devem ser resolvidas por meio de procedimentos numéricos iterativos. Os métodos iterativos mais usados na TRI são o algoritmo "Newton-Raphson" e o método "Scoring" de Fischer, além de outros, como os métodos de quadratura e o algoritmo "Esperança e Maximização" [3].

Os três parâmetros apresentados e medidos no modelo são: o parâmetro "a", representado pela curvatura no ponto de inflexão, onde a curva corta a linha que representa a probabilidade de $50 \%$. A inclinação da curva é proporcional a sua discriminação, ou seja, quanto mais íngreme a curva mais discriminativo é o estímulo. O parâmetro " $b$ " mede a dificuldade do estímulo sendo representado pela distância na linha do X (abscissa), que corresponde ao ponto determinado pela perpendicular que vem do ponto de inflexão da curva. O parâmetro "c" afere o acerto casual, no qual um sujeito com baixa proficiência venha responder corretamente ao estímulo, definido pela distância entre o ponto zero da abscissa até o ponto onde a curva corta a ordenada [2].

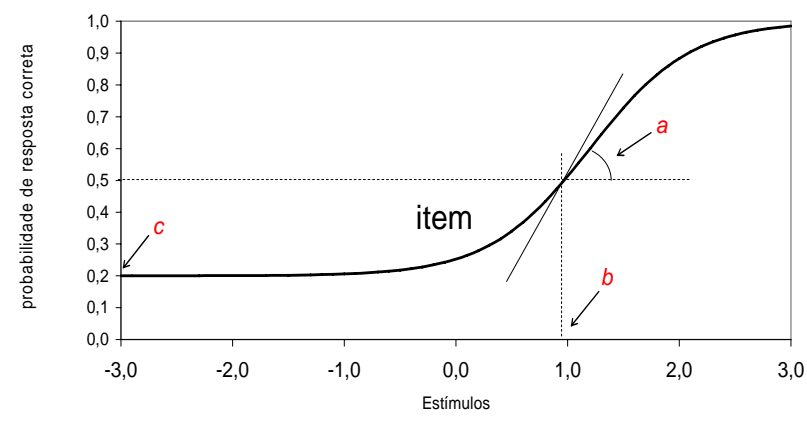

Figura 1. Função da Curva Característica do Item

\section{Modelo Computacional}

O modelo computacional PPG é uma arquitetura genérica para geração de perfis. $\mathrm{O}$ modelo funciona como motor para prospecção dos níveis de habilidades e ou comportamentos na forma de itens e/ou estímulos. Esses itens e/ou estímulos representam o núcleo do gerador com seus valores recebidos dos sistemas externos, servindo como base para os cálculos de prospecção do perfil.

O PPG interage somente com os sistemas externos, não tendo nenhum contato com os usuários desses sistemas. O gerador processa e retorna aos sistemas as informações sobre os perfis dos usuários, ou seja, seus níveis de comportamento ou habilidade devidamente identificados e prospectados através do modelo matemático TRI. Os itens e ou estímulos a serem utilizados e a forma como eles serão aplicados são definidos por especialistas das áreas específicas do conhecimento, ou seja, psicólogos e/ou pedagogos.

A Figura 2 descreve a integração do PPG com os sistemas externos, bem como a interação do especialista entre o PPG e os sistemas externos, onde: (a) sistemas externos: representa qualquer tipo de sistema externo ao PPG, desde sistemas educacionais, entretenimento ou recrutamento e seleção, podendo também ser 
simplesmente uma prova de um professor de matemática, um questionário de um psicólogo ou um sistema de educação a distância que deseja utilizar o PPG; (b) camada de acoplamento: o propósito de criar uma camada de acoplamento aos sistemas externos se explica pelo fato da intenção de reduzir as mudanças nesses sistemas, procurando aumentar a funcionalidade do PPG e sua adaptação aos sistemas externos; (c) módulo do especialista: o especialista possui uma interface direta com o PPG e o(s) sistema(s) externo(s), pois necessita realizar intervenções nas duas camadas do processo de utilização do PPG; (d) dados para prospecção: os dados recebidos pela camada de acoplamento dos sistemas externos são validados e enviados ao PPG; (e) PPG: responsável por receber os dados da camada de acoplamento e prospectar os perfis dos usuários dos sistemas externos; (f) dados prospectados: após o processamento interno do PPG para prospectar o perfil do usuário, o mesmo é devolvido aos sistemas externos passando pela camada de acoplamento.

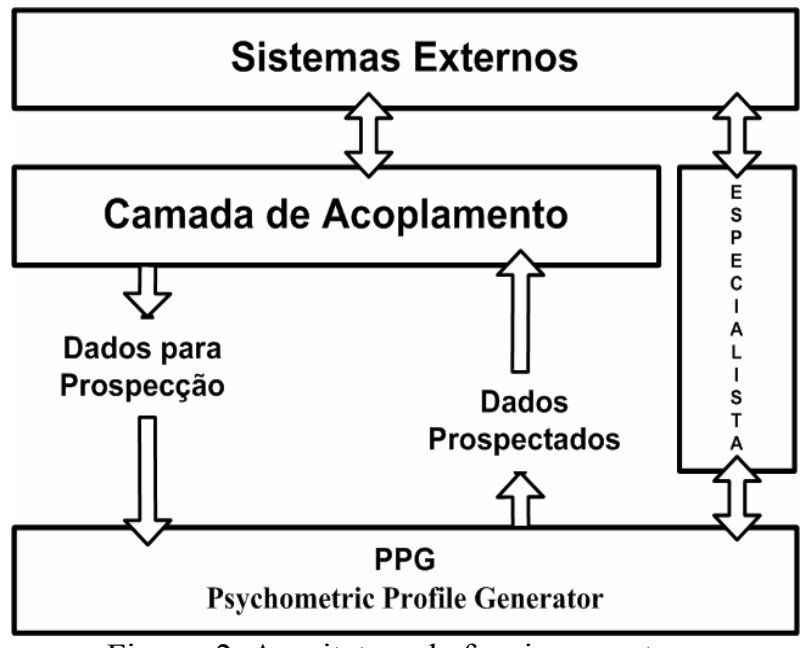

Figura. 2. Arquitetura de funcionamento.

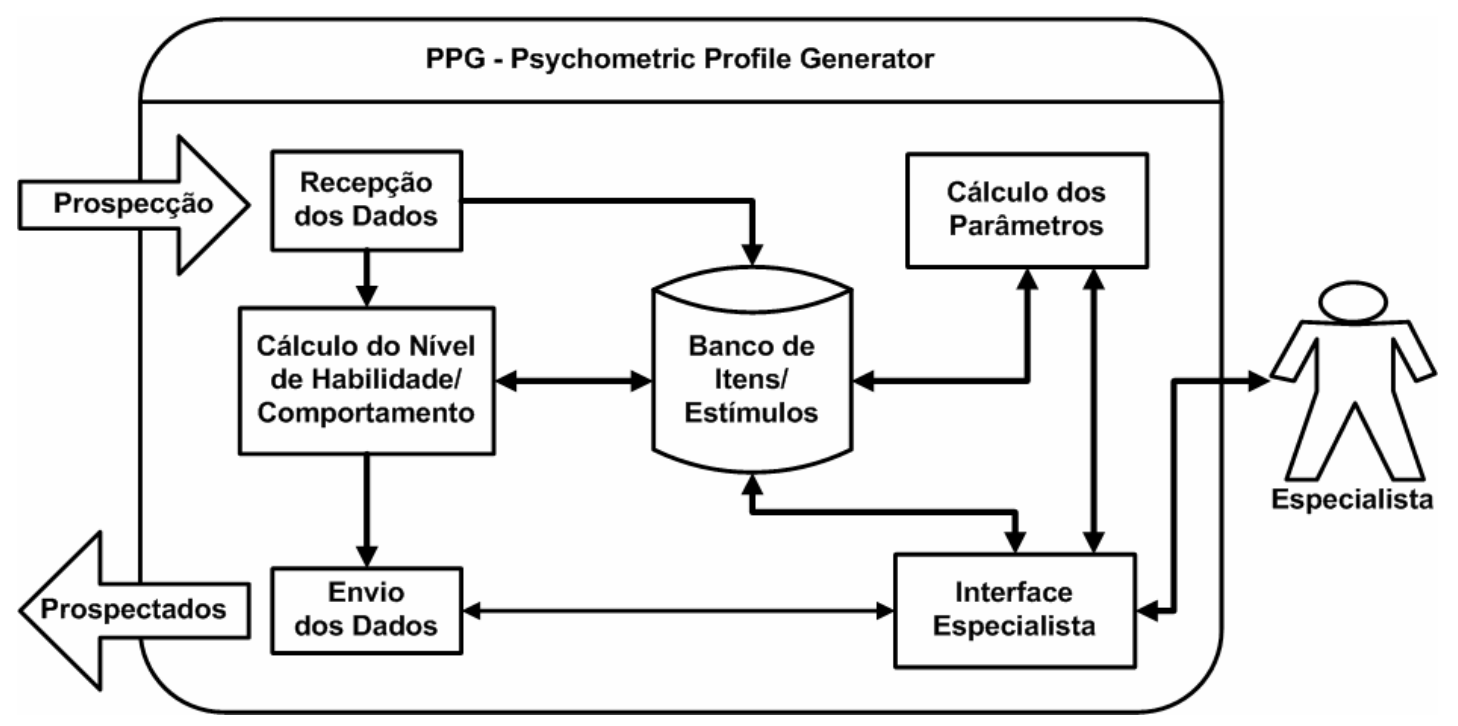

Figura. 3. Organização Interna do PPG.

A Figura 3 apresenta a estrutura interna do PPG, suas interfaces com os sistemas externos e seu fluxo de processamento do perfil dos usuários.

O PPG é composto por cinco módulos e um banco de dados: (a) módulo "Recepção dos dados": responsável pela recepção dos dados da camada de acoplamento, gravação dos itens ou estímulos e acionamento do módulo "Calcular Nível de Habilidade/Comportamento"; (b) módulo "Cálculo dos Parâme- tros": realiza os cálculos inerentes a prospecção dos parâmetros "a" (discriminação), "b" (dificuldade) e "c" (acerto casual) através do modelo matemático TRI; (c) módulo "Interface Especialista": fornece uma interface para o especialista cadastrar os sistemas externos, os usuários, as habilidades ou comportamentos e seus respectivos itens e ou estímulos. O módulo também possibilita ao especialista visualizar as representações gráficas dos itens e ou estímulos. Viabiliza ainda, consultar os parâmetros prospectados por itens (habilidade) 
e ou estímulos (comportamento); (d) módulo "Envio dos dados": gera e envia os dados devidamente identificados; (e) módulo "Cálculo do Nível de Habilidade/Comportamento": responsável por determinar o nível de habilidade ou comportamento, utilizando a equação 2 que descreve, conforme Santos [10], o princípio da mediana como uma maneira de calcular o nível de habilidade ou comportamento de um avaliado.

$$
\bar{X}=\frac{\sum_{i=1}^{n}\left(1-P(\theta)_{i}\right) R_{i}}{n} \quad(i=1,2, \ldots \ldots, n)
$$

probabilidades $\operatorname{Pi}(\theta)$ de um avaliado responder corretamente determinado item $i$; (2) a variável $R$ representa o valor da resposta do avaliado, sendo 0 para respostas incorretas e 1 para respostas corretas, dessa maneira as respostas incorretas não participarão do cálculo; (3) $n$ representa o total de itens que compõem a habilidade em questão.

A camada de acoplamento foi implementada utilizando a linguagem Java. O PPG recebe um arquivo XML seguindo o padrão mostrado na figura 4 através de conexões via sockets na porta 3636.

A equação 2 contém: (1) um somatório de todas as

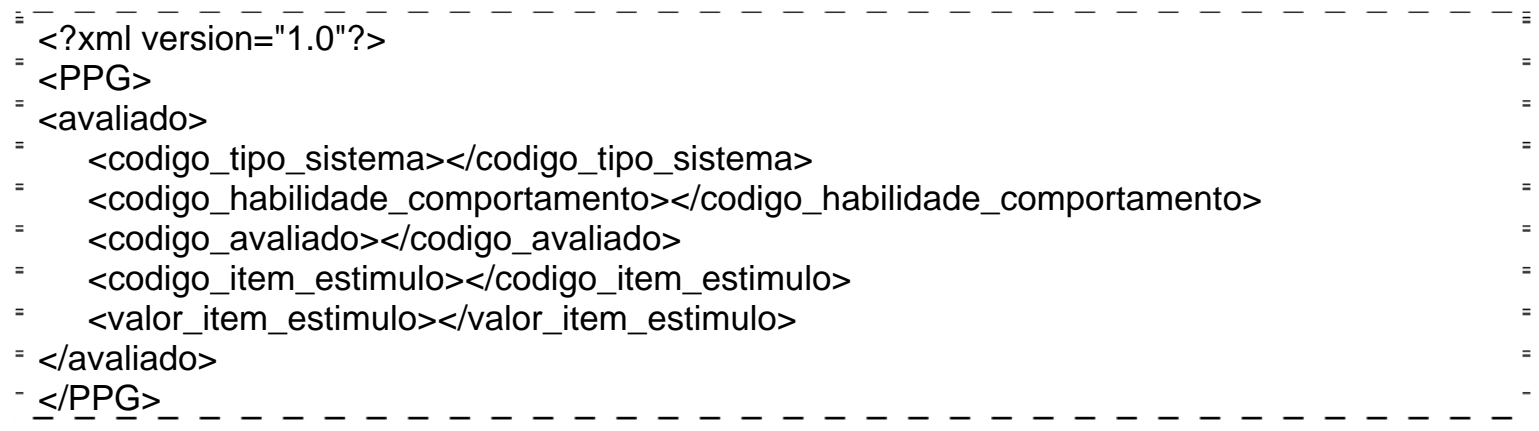

Figura 4 - Estrutura do arquivo XML dos dados para prospecção

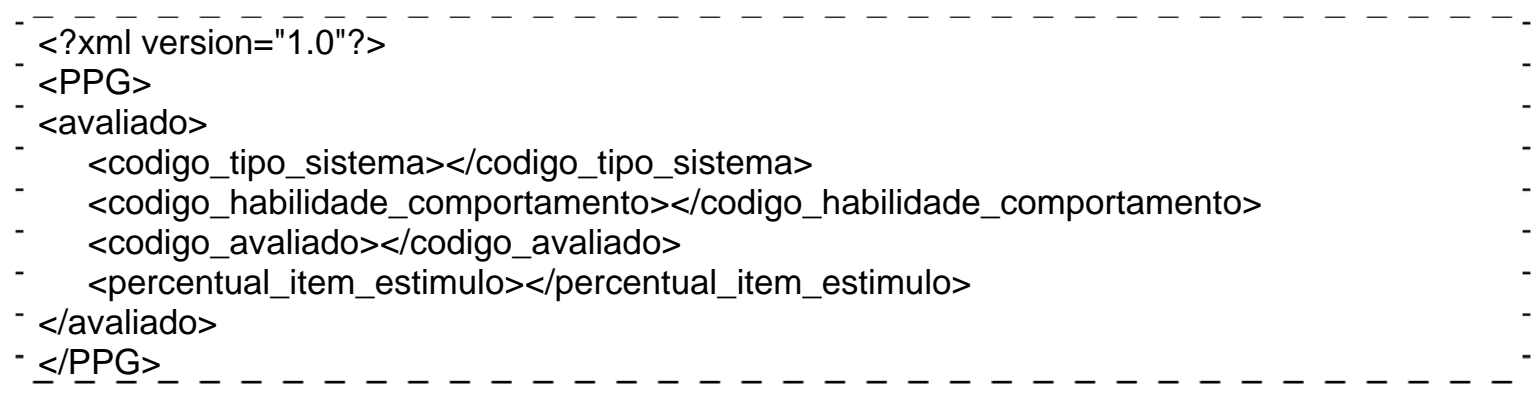

Figura 5 - Estrutura do arquivo XML dos dados prospectados

Os dados são tratados, validados e gravados no banco do PPG para realização dos cálculos dos parâmetros. O retorno dos níveis de habilidades ou comportamentos acontece no mesmo canal de comunicação aberto pelo sistema externo, através do arquivo XML descrito na figura 5 .

A utilização do PPG possui três fases distintas para construção de uma habilidade ou comportamento: (1) a primeira fase é a definição da habilidade ou comportamento a ser medido; (2) a segunda é a construção de itens que mensurem uma determinada habilidade ou estímulos que afiram um comportamento específico, juntamente com a coleta desses itens ou estímulos, compreendendo as análises gráficas e parâmetros disponibilizados pelo modelo matemático TRI; (3) a terceira fase é a utilização do PPG de forma autônoma. Esse ciclo entre as fases deve ser respeitado, pois depende dele a construção de itens ou estímulos que realmente mensurem um determinado perfil. 


\section{PPG aplicado em Jogos Educacio- nais}

A proposta de utilizar um jogo em RPG educacional como cenário para validar o PPG se consolida em função da facilidade da criação de personagens, ações e roteiros conforme as necessidades de mensuração dos especialistas (pedagogos ou psicólogos) em apurar um ou mais tipos de comportamentos ou habilidades dos avaliados.

No cenário proposto para validação estão agregadas as questões de mensuração de habilidades e comportamentos, nas quais os jogadores, além de interagirem com os outros personagens, interagem com diversos ambientes que mesclam o virtual com o real, conforme figura 6 .

A figura 6 apresenta as principais telas do jogo. $\mathrm{O}$ cenário escolhido foi $\mathrm{o}$ ambiente de uma escola. $\mathrm{O}$ aluno inicia o jogo no pátio da escola, devendo escolher um ambiente para interagir. Caso não ocorra a escolha, um personagem (nesse caso na figura de um homem) irá avisar sobre a existência dos ambientes.

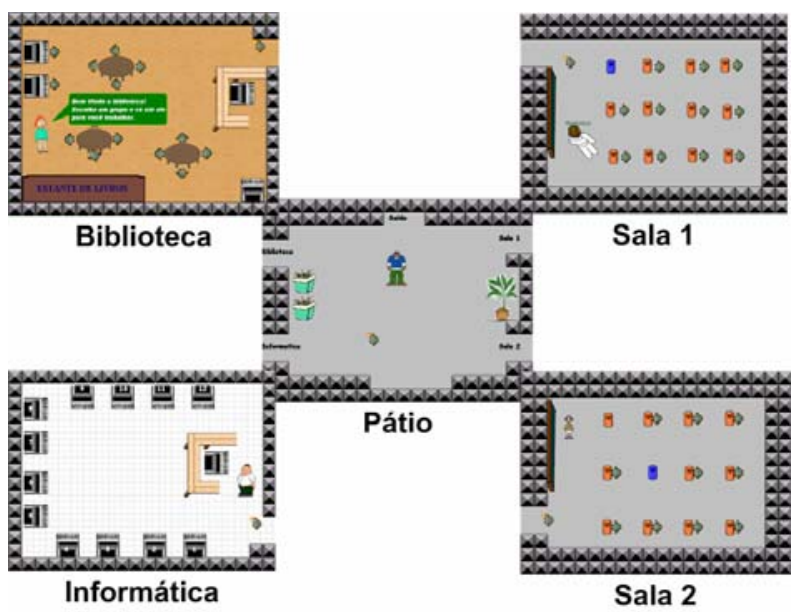

Figura. 6. Telas do Jogo

Os ambientes são duas salas de aula, um laboratório de informática e a biblioteca. Nas salas de aulas encontram-se um professor e os demais colegas. O laboratório de informática possui doze computadores e um professor. A biblioteca possui três computadores, três mesas, uma estante de livros e um bibliotecário.

O avaliado ao entrar nos ambientes deverá responder às questões apresentadas, não sendo possível sair do ambiente até responder todas as questões. Todas as questões utilizadas fazem parte da prova Brasil de 2007.

A habilidade que foi tratada pelo jogo foi obtida no site do INEP ${ }^{1}$ (Instituto Nacional e Estudos e Pesquisas) e também no site da Prova Brasil ${ }^{2}$. A Prova Brasil, criada em 2005 a partir da necessidade de tornar a avaliação mais detalhada, é censitária. Por esta razão, expande o alcance dos resultados porque oferece dados, não apenas para o Brasil e unidades da Federação, mas também para cada município e escola participante. A Prova Brasil avalia todos os estudantes da rede pública urbana de ensino, de $5^{\mathrm{a}}$ à $9^{\mathrm{a}}$ série do Ensino Fundamental de 9 anos.

Ao completar a $5^{\text {a }}$ série do Ensino Fundamental, o aluno deve possuir algumas habilidades relacionadas ao espaço e forma que serão aferidas pela Prova Brasil. Nesse sentido, o jogo proposto auxilia os educadores a aferirem as habilidades, pois o jogo educacional irá interagir com o PPG e poderá tomar caminhos que facilitem o aprendizado, fornecendo, assim, informação sobre o nível dessa habilidade.

Esse contexto de fornecer ferramentas que auxiliem o educador a tomar decisões durante o processo educacional é muito importante, pois o educador terá tempo hábil para retomar pontos ainda não bem sedimentados pelos alunos e fornecerá uma maneira lúdica de medir as habilidades.

\section{Resultados}

$\mathrm{O}$ número de alunos que participaram desse experimento foi de cento e treze, divididos em três turmas de aproximadamente 35 alunos com idade média de 11 anos. O local utilizado foi o laboratório de uma escola particular do Estado do Rio Grande do Sul, na cidade de Gravataí, com equipamentos Intel 1,7 GHz, 256Mb de memória e $64 \mathrm{Mb}$ memória de vídeo compartilhada. Não foi mencionado aos avaliados que participariam de uma avaliação, mas sim que estariam participando de um jogo. Essa decisão de não avisar fez parte da estratégia do especialista, pois o mesmo acreditava que sem a pressão de uma avaliação os alunos estariam respondendo às questões com maior tranqüilidade. Também não foi estipulado tempo para os alunos responderem às questões.

O tempo médio gasto por um aluno foi de 11 minutos e 44 segundos para responder 10 itens de habilidade e 5 estímulos de comportamento. $\mathrm{O}$ avaliado que utilizou mais tempo, valeu-se de 24 minutos e 7 segundos. Já o avaliado com menor tempo realizou o teste em 4 minutos e 30 segundos.

O gráfico na figura 7 e a tabela 1 apresentam o comportamento dos itens após os cento e treze alunos jogarem. Observa-se que os itens 2, 5, 7 e 10 obtiveram

\footnotetext{
${ }^{1}$ Disponível em: $<$ http://www.inep.gov.br/>.

${ }^{2}$ Disponível em: $<$ http://provabrasil.inep.gov.br $>$.
} 
valores altos, no que se refere ao acerto casual, variando de 0,1780 a 0,5550 mostrando os itens que não

conseguiram calibrar junto ao grupo aplicado.

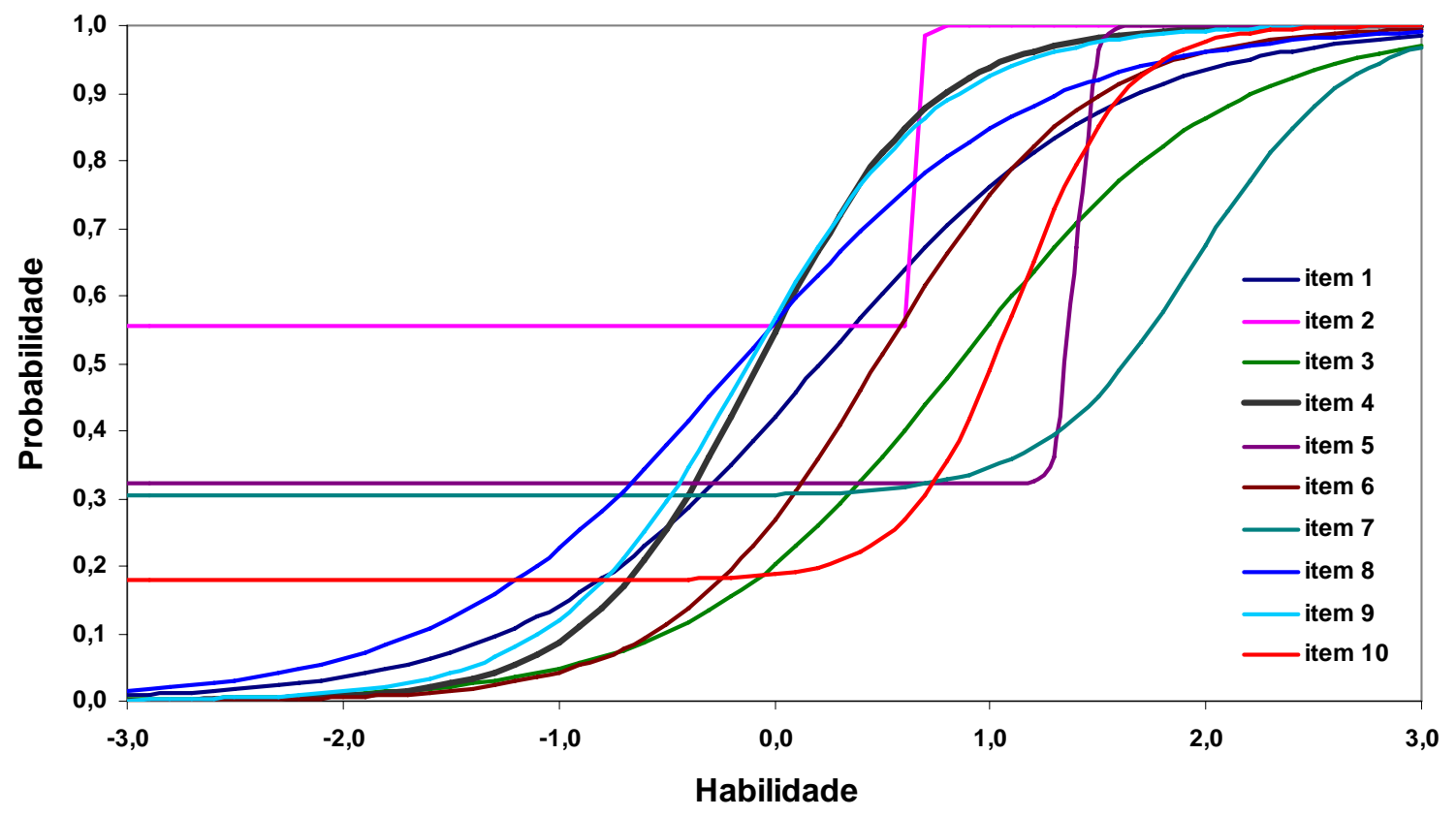

Figura. 7. Curva característica dos itens aplicados nos alunos da $5^{\text {a }}$ série de 9 anos

Ao analisar a tabela 1 com os parâmetros prospectados ao finalizar o recebimento das respostas dos cento e treze alunos pelo PPG, observa-se valores discrepantes na coluna discriminação nos mesmos itens que apresentaram acerto casual alto. Conclui-se que esses itens apresentam uma classificação relevante entre os avaliados, pois os avaliados que acertaram somente esses itens foram classificados como avaliados que escolhem seus itens ao acaso e não possuem nenhuma proficiência na habilidade desejada.

\begin{tabular}{c|c|c|c|c}
\hline Itens & Discriminação & Dificuldade & Acerto Casual & P \\
\hline 1 & 0,8740 & 0,2160 & 0,0000 & 0,5617 \\
\hline 2 & 83,8410 & 0,6760 & 0,5550 & 0,5550 \\
\hline 3 & 0,9480 & 0,8520 & 0,0000 & 0,4173 \\
\hline 4 & 1,4890 & $-0,0740$ & 0,0000 & 0,7015 \\
\hline 5 & 16,6730 & 1,3980 & 0,3220 & 0,3220 \\
\hline 6 & 1,2380 & 0,4770 & 0,0000 & 0,5071 \\
\hline 7 & 1,6920 & 1,9530 & 0,3030 & 0,3579 \\
\hline 8 & 0,8670 & $-0,1630$ & 0,0000 & 0,6399 \\
\hline 9 & 1,3300 & $-0,1170$ & 0,0000 & 0,6944 \\
\hline 10 & 2,3480 & 1,1240 & 0,1780 & 0,3323 \\
\hline
\end{tabular}

Tabela 1. Parâmetros prospectados na $5^{\text {a }}$ série de 9 anos

O cálculo do nível de habilidade ou comportamento apresentado na equação 2 torna esses itens com peso menor em comparação aos demais. Essa análise utilizase dos casos coletados do relatório apresentado na tabela 2 com uma amostragem dos alunos classificados por série, turma, nome fictício, suas respostas e o nível de habilidade prospectada.

\begin{tabular}{|l|l|l|r|r|r|r|r|r|r|r|r|r|c|}
\hline Série & Turma & Nome & R1 & R2 & R3 & R4 & R5 & R6 & R7 & R8 & R9 & R10 & NÍVEL \\
\hline 5 & A & ALUNO 1 & 1 & 1 & 1 & 1 & 0 & 1 & 1 & 1 & 1 & 1 & 93,67 \\
\hline 5 & B & ALUNO 8 & 1 & 1 & 1 & 1 & 1 & 1 & 0 & 1 & 1 & 1 & 92,97 \\
\hline 5 & B & ALUNO 9 & 1 & 1 & 1 & 1 & 0 & 0 & 0 & 1 & 1 & 0 & 70,15 \\
\hline 5 & A & ALUNO 2 & 1 & 0 & 1 & 0 & 0 & 1 & 1 & 1 & 1 & 1 & 68,98 \\
\hline 5 & C & ALUNO 1 & 1 & 1 & 0 & 1 & 0 & 0 & 0 & 0 & 1 & 1 & 55,90 \\
\hline 5 & A & ALUNO 3 & 1 & 0 & 1 & 1 & 1 & 0 & 1 & 0 & 0 & 1 & 52,91 \\
\hline 5 & A & ALUNO 4 & 0 & 1 & 1 & 1 & 0 & 0 & 0 & 1 & 0 & 0 & 45,46 \\
\hline 5 & A & ALUNO 5 & 0 & 1 & 1 & 0 & 0 & 1 & 0 & 1 & 0 & 0 & 41,64 \\
\hline
\end{tabular}




\begin{tabular}{|l|l|l|l|l|l|l|l|l|l|l|l|l|l|}
5 & B & ALUNO 10 & 0 & 1 & 1 & 0 & 0 & 0 & 0 & 0 & 1 & 1 & 39,28 \\
\hline 5 & A & ALUNO 6 & 1 & 1 & 0 & 0 & 0 & 0 & 0 & 0 & 0 & 0 & 21,94 \\
\hline 5 & A & ALUNO 7 & 0 & 0 & 0 & 0 & 1 & 0 & 1 & 0 & 0 & 0 & 13,36 \\
\hline
\end{tabular}

Tabela 2. Tabela com uma amostragem dos resultados da $5^{\text {a }}$ série de 9 anos

Algumas análises fazem-se necessárias: (a) os alunos 1 e 8 erraram somente um item cada um, mas suas notas apresentam 0,70 pontos de diferença. Em provas normais suas notas seriam iguais, não tendo diferença alguma; (b) os alunos 9 e 2 apresentam um diferença maior, sendo de 1,16 pontos, observa-se que o aluno com maior nível de habilidade obteve 70,15 errando um questão a mais que o aluno 2 , já o aluno com menor nível de habilidade obteve 68,98 errando uma questão a menos em relação ao aluno 9 . O aluno com maior nível de habilidade errou quatro itens, e o aluno com menor nível errou somente três itens. O nível de habilidade está atrelado ao nível de dificuldade, discriminação e acerto casual de cada item que apresenta probabilidade de acerto menor e conseqüentemente peso maior ao avaliado ao calcular o nível de habilidade; (c) observase discrepância entre os alunos 6 e 7, uma vez que os dois acertam somente dois itens e suas notas apresentam 8,58 pontos de diferença; (d) verifica-se que cada item contribui com seus valores para o cálculo do nível de habilidade dos avaliados. O experimento de avaliar cento e treze alunos mostrou-se válido para calcular o nível de habilidade proposto pelo PPG, sendo também utilizado na prática pelo especialista para trabalhar com seus educadores, cada item e consequentemente os reflexos ao constatar que alguns conteúdos trabalhados ainda apresentam níveis de dificuldade e acerto casual alto entre os avaliados.

\subsection{Análise Progressiva dos Resultados}

A análise progressiva foi realizada após o PPG receber do jogo 79 respostas de avaliados, com o intuito de verificar a variação da probabilidade de acerto de cada item em relação ao aumento do número de avaliados. Foram realizados os cálculos dos parâmetros a cada entrada de 5 avaliados e suas respectivas respostas, calculou-se a probabilidade de acerto de cada item, repetindo-se 7 vezes esse processo.

A figura 8 apresenta a análise progressiva, onde o eixo da linha do $\mathrm{X}$ (abscissa) representa o número de avaliados. O eixo da coluna $\mathrm{Y}$ (ordenada) a probabilidade de acerto de um determinado item. A cada cinco alunos que terminavam suas jogadas, os resultados eram somados com os anteriores e entravam nos cálculos de prospecção dos parâmetros de dificuldade, discriminação e acerto casual.

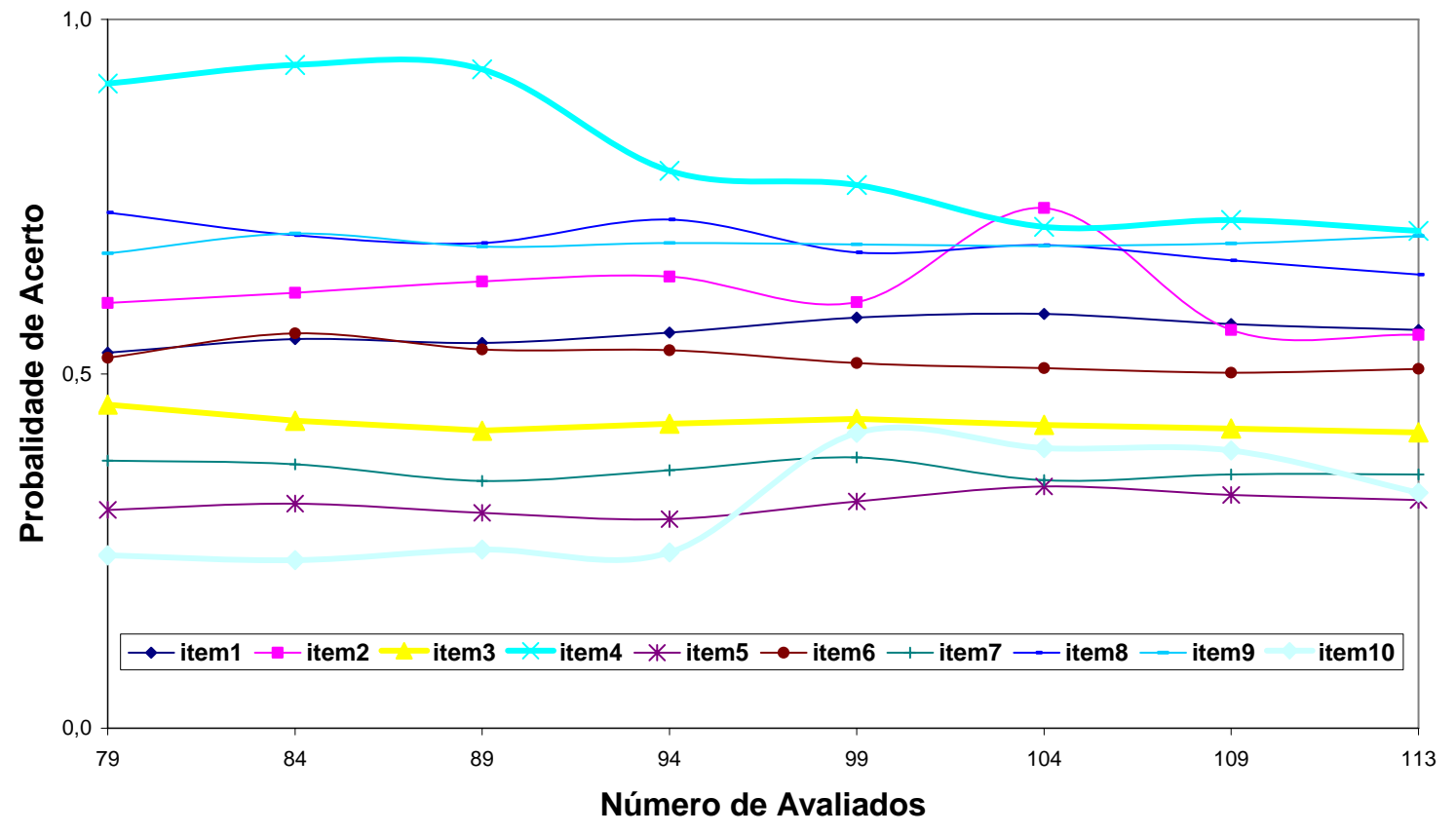

Figura 8 - Gráfico da evolução da probabilidade de acerto ao utilizar o PPG 


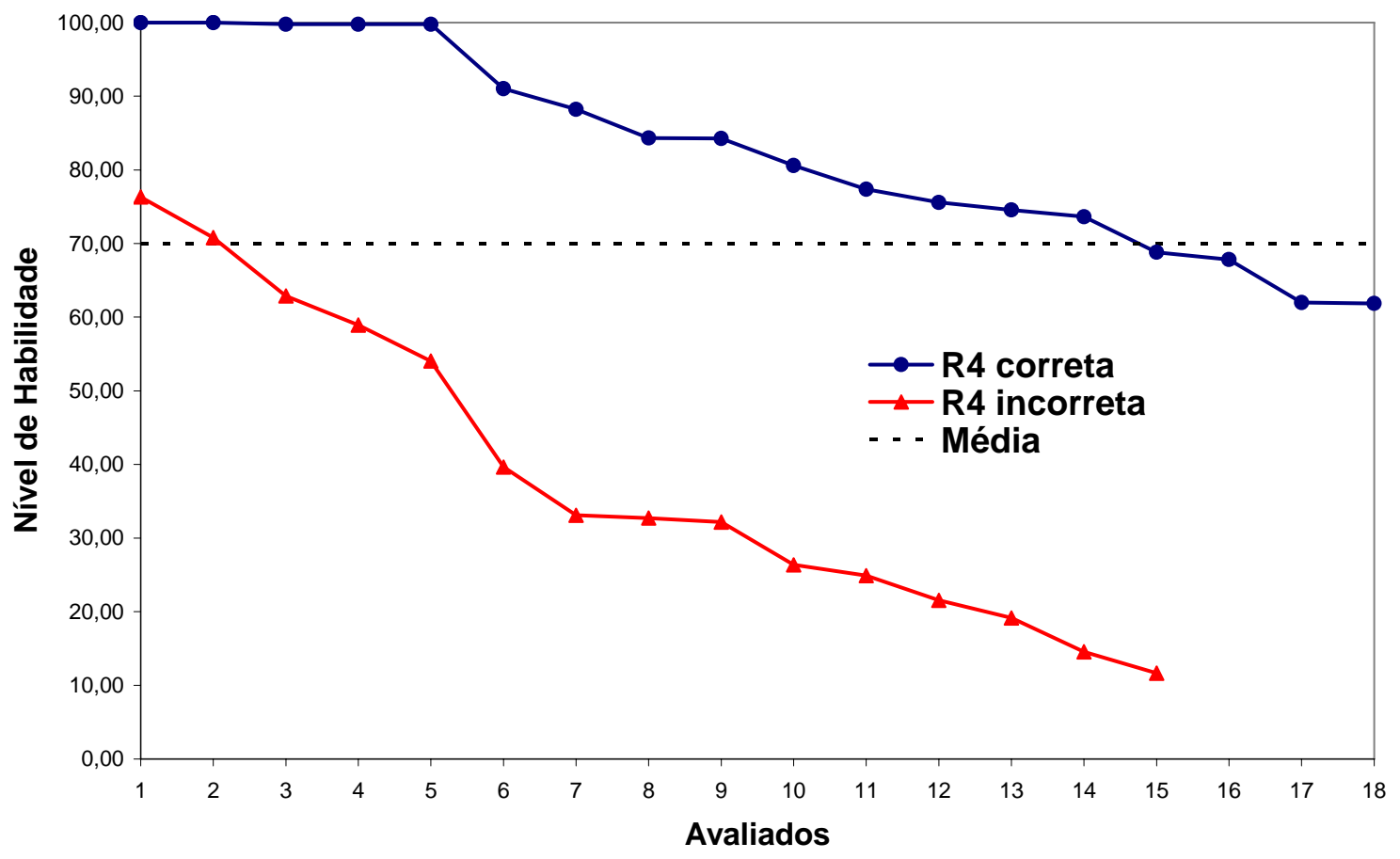

Figura 9 - Gráfico de adaptação do jogo em função do nível de habilidade

A variação média encontrada entre os setenta e nove avaliados e os cento e treze foi de 1,74 por cento e o desvio padrão de 1,34 .

Observa-se no gráfico da figura 8 uma tendência de estabilização das curvas. Essa tendência em manter-se constante demonstra um possível ponto de parada nos cálculos dos parâmetros, viabilizando a criação de parâmetros constantes nas fórmulas utilizadas para prospecção de um perfil.

Em analogia a redes neurais, pode-se afirmar que os itens foram treinados e validados para o grupo pretendido. Observa-se uma tendência em manter constantes os parâmetros, gerando uma regressão logística dos dados prospectados.

\subsection{Resultados da Adaptação do Jogo}

A terceira fase de utilização do PPG é a fase autônoma, ou seja, essa fase não necessita da intervenção do especialista. O PPG fornece o nível de habilidade ou comportamento de forma automática ao sistema externo. O sistema externo poderá ou não utilizar essa informação para adaptar-se aos seus usuários.

Através da interação de um sistema externo e o PPG, fecha-se um ciclo de utilização, composto em três fases, todas ligadas ao sistema externo. O papel do especialista nas duas primeiras fases viabiliza a fase autônoma do PPG, ou seja, terceira fase. O especialista deverá interagir com o sistema externo (jogo), e o PPG, definindo as habilidades ou comportamentos, coletando e cadastrando os dados no PPG. A validação dos dados cadastrados ocorre na segunda fase de utilização.

A segunda fase do jogo foi desenvolvida pensando em utilizar as informações fornecidas pelo PPG e realizar a adaptação do jogo em função do nível de habilidade do avaliado. Com base nos 113 alunos que jogaram a primeira versão do jogo, foi possível determinar o nível de dificuldade de cada questão.

O experimento realizado foi aplicado em alunos da turma da $5^{\text {a }}$ série $\mathrm{D}$ de 9 anos, compreendendo 33 alunos. O especialista de posse das informações recebidas pelo PPG na segunda fase definiu: (1) a questão de número 4 (R4) como sendo a questão de nível médio de dificuldade; (2) todo aluno que errar a R4 deverá responder todas as questões do jogo; (3) o aluno que acertar a R4 não responderá as questões fáceis; (4) as questões fáceis foram colocadas no laboratório de informática; (5) as demais questões na biblioteca e sala $2 ;(6)$ a questão R4 ficou alocada na sala 1; (7) ficou bloqueado o acesso aos demais ambientes, até o jogador entrar na sala 1 e responder a $\mathrm{R} 4$.

A figura 9 apresenta o nível de habilidade em ordem decrescente por avaliado. O desempenho do melhor ao pior aluno separando-os em dois grupos. $\mathrm{O}$ 
grupo que acertou a questão R4 e o grupo que errou a

Observa-se no gráfico da figura 9 que os avaliados que erraram a questão R4 obtiveram o desempenho inferior aos que acertaram a questão R4. Uma separação notória entre os dois grupos tendo como base a média mínima exigida de $70 \%$ do nível de habilidade.

Do universo de 33 alunos submetidos à segunda fase do jogo, 15 alunos erraram a questão R4 e somente 2 obtiveram um nível de habilidade acima de $70 \%$. Já os 18 alunos que acertaram a questão R4, somente 4 obtiveram nível de habilidade abaixo de $70 \%$.

A adaptação proposta pelo jogo utilizando as informações fornecidas pelo PPG apresentou-se satisfatória, pois dos alunos que erraram a questão R4, 87\% ficaram abaixo da média e $13 \%$ acima da média. No entanto os alunos que acertaram a questão R4, um total de $78 \%$ ficaram acima da média e $22 \%$ abaixo da média. Em resumo a menor média dos alunos que acertaram R4 foi $61,85 \%$ e a maior média dos que erraram R4 foi $76,31 \%$. questão

R4.

\subsection{Utilização do PPG para Analisar A- lunos de $4^{\mathrm{a}}$ série}

No processo de validação do PPG foi aplicado o jogo aos alunos de $4^{\mathrm{a}}$ série, ou seja, uma série inferior à definida pelo especialista como público alvo para verificar o nível de habilidade. Mesmo sabendo que estaríamos colocando esses avaliados em situação de desconforto, pois não saberiam solucionar ou até mesmo tratar os problemas apresentados, o especialista considerou interessante ter esses resultados apurados para trabalhar com seus educadores os resultados obtidos.

A Figura 10 apresenta a curva prospectada após a aplicação em cento e onze alunos, com um grande número de questões com acerto casual alto. Em contrapartida verifica-se a aplicabilidade do modelo matemático utilizado pelo PPG, pois os resultados demonstraram que esses itens não seriam calibrados para esse universo de avaliados.

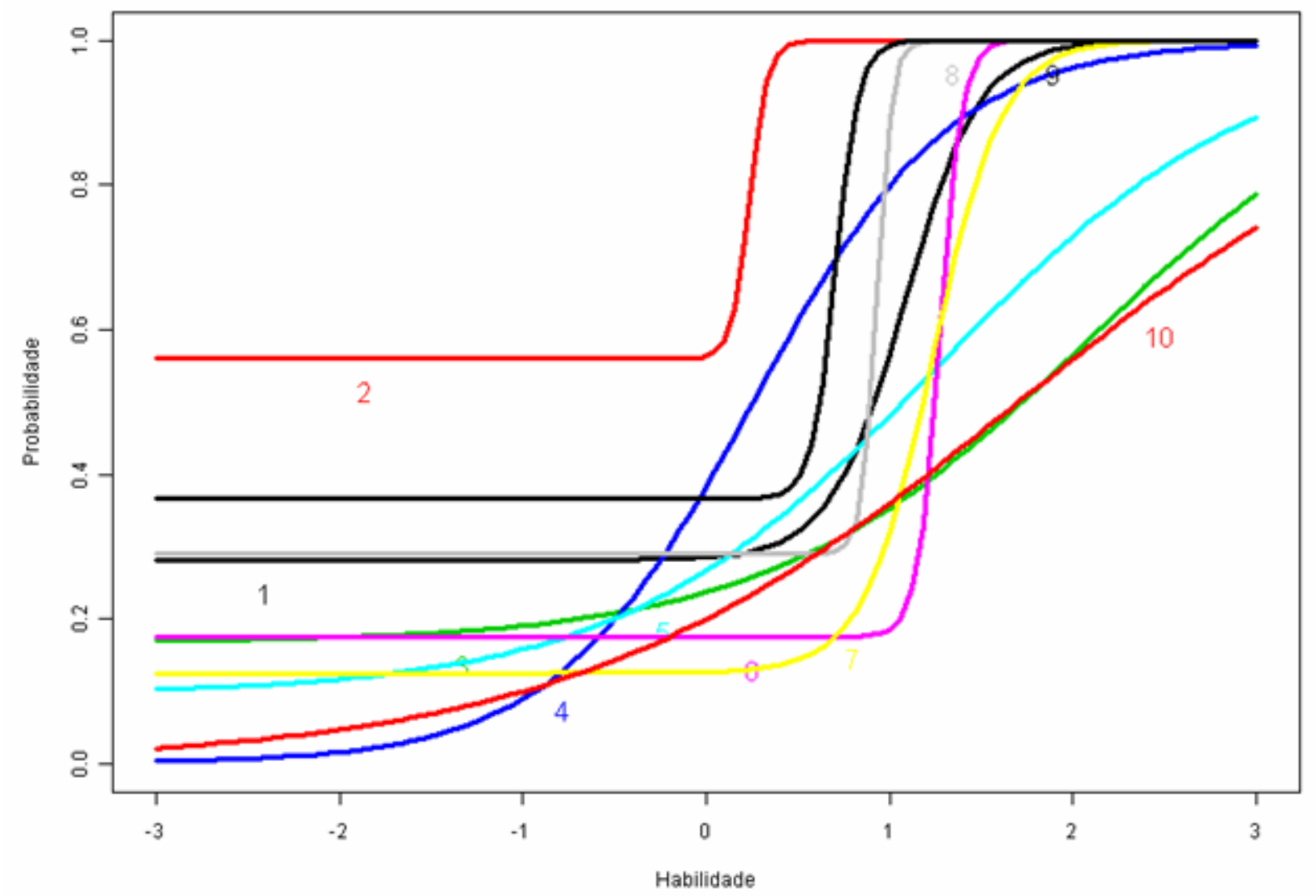

Figura 10 - Gráfico dos itens aplicados aos alunos da $4^{\mathrm{a}}$ série

Deixando abertura para utilizações onde mesmo o especialista aplicando avaliações não projetadas para o nível de escolaridade, ou até mesmo intelectuais do avaliado, o PPG disponibilizou a informação correta do 
nível de habilidade dos avaliados.

A Tabela 3 descreve ao especialista esses valores altos de acerto casual, com o maior valor em 0,5600 por cento de probabilidade dos itens estarem sendo escolhido na casualidade.

\begin{tabular}{rcccc}
\hline Itens & Discriminação & Dificuldade & Acerto Casual & P $\theta$ \\
1 & 4,8970 & 1,0860 & 0,2820 & 0,3205 \\
2 & 20,0380 & 0,2360 & 0,5600 & 0,9978 \\
3 & 1,1560 & 2,0770 & 0,1670 & 0,2828 \\
4 & 1,8550 & 0,2580 & 0,0010 & 0,6108 \\
5 & 1,1550 & 1,2610 & 0,0960 & 0,3612 \\
6 & 16,1240 & 1,2720 & 0,1750 & 0,1750 \\
7 & 5,2860 & 1,2350 & 0,1250 & 0,1426 \\
8 & 24,4700 & 0,9310 & 0,2910 & 0,2910 \\
9 & 15,6540 & 0,7040 & 0,3670 & 0,3919 \\
10 & 0,8130 & 1,7070 & 0,0000 & 0,2726 \\
\hline
\end{tabular}

Tabela 3 - Parâmetros prospectados na $4^{\mathrm{a}}$ série

Foi entregue para o especialista uma listagem com todos os cento e onze (111) alunos descrevendo série, turma, nome, as respostas indicando 0 (zero) como erro e 1 (um) como acerto do item e o nível de habilidade do aluno. A tabela 3 mostra alguns casos identificados com nomes fictícios, mas com valores reais.

\begin{tabular}{|r|l|l|r|r|r|r|r|r|r|r|r|r|c|}
\hline Série & Turma & Nome & R1 & R2 & R3 & R4 & R5 & R6 & R7 & R8 & R9 & R10 & Nível Habilidade \\
\hline 4 & A & Aluno 19 & 1 & 1 & 1 & 1 & 1 & 1 & 0 & 1 & 1 & 1 & 96,29 \\
\hline 4 & B & Aluno 42 & 1 & 1 & 0 & 1 & 1 & 1 & 1 & 1 & 1 & 0 & 85,56 \\
\hline 4 & B & Aluno 68 & 1 & 1 & 0 & 1 & 0 & 0 & 1 & 1 & 0 & 0 & 61,43 \\
\hline 4 & A & Aluno 13 & 1 & 1 & 0 & 0 & 0 & 1 & 1 & 1 & 1 & 0 & 60,29 \\
\hline 4 & B & Aluno 63 & 0 & 1 & 0 & 0 & 0 & 0 & 0 & 1 & 0 & 0 & 33,51 \\
\hline 4 & B & Aluno 45 & 0 & 1 & 1 & 0 & 0 & 0 & 0 & 0 & 0 & 0 & 33,29 \\
\hline 4 & B & Aluno 40 & 0 & 0 & 1 & 0 & 1 & 0 & 0 & 0 & 0 & 1 & 23,83 \\
\hline 4 & C & Aluno 78 & 0 & 0 & 0 & 1 & 0 & 1 & 0 & 0 & 0 & 0 & 20,43 \\
\hline 4 & A & Aluno 28 & 0 & 0 & 0 & 0 & 1 & 0 & 0 & 0 & 1 & 0 & 19,58 \\
\hline
\end{tabular}

Tabela 4 - Amostra do relatório fornecido ao especialista

Observa-se que os alunos 78 e 28 computaram o mesmo número de acertos, mas acertando e errando itens diferentes, seus níveis de habilidade também são diferentes. Já os alunos 68 e 13 apresentam níveis de habilidade próximos com diferença de 1,14. Mas vale observar que o aluno 68 acertou somente a metade das questões, obtendo nível de habilidade maior que seu colega o aluno 13 que acertou seis questões, obtendo nível de habilidade menor que o colega que acertou somente cinco questões.

Em resumo, não é o número de questões acertadas que definem o nível de habilidade de um avaliado, mas sim o número de questões acertadas com maior nível de dificuldade e por conseqüência com menor probabilidade de acerto. Nesse caso estamos diante de um sistema de avaliação mais justo, pois quanto maior sua habilidade maior sua nota. Precisamos de sistemas que avaliem o aluno como um todo, fornecendo ao educador uma ferramenta que propicie avaliar o nível de suas questões perante o grupo de avaliados.

Mesmo esse experimento nos mostrando que os itens possuem um nível alto de acertos casuais, o mesmo serviu para aferir o PPG e colocar em prática o modelo matemático TRI e o modelo computacional do PPG.

\section{Trabalhos Relacionados}

A Tabela 5 apresenta os três trabalhos relacionados pesquisados, ou seja: (1) testes com pilotos norteamericanos na identificação de aeronaves, aplicando o mesmo teste na forma de papel e de um jogo computadorizado, apurando através da correlação estatística a 
viabilidade de ambos [4]; (2) teste com idosos, em três tipos de equipamentos (mouses, touch screen e tabletwith-stylus) juntamente com um teste de detecção dos níveis das funções cognitivas MMSE (Mini-Mental State Examination) e nível social, para traçar o perfil do idoso e sua relação com as tecnologias [5];

\begin{tabular}{ccccc}
\hline Utilização/Testes/Geração & $\begin{array}{c}\text { Pilo- } \\
\text { tos }\end{array}$ & $\begin{array}{c}\text { Ido- } \\
\text { sos }\end{array}$ & Jogos Massivos & $\begin{array}{c}\text { PP } \\
\text { G }\end{array}$ \\
\hline Perfil de Usuários & $\mathrm{X}$ & $\mathrm{X}$ & & $\mathrm{X}$ \\
\hline Inferência Estatística & $\mathrm{X}$ & $\mathrm{X}$ & $\mathrm{X}$ & $\mathrm{X}$ \\
\hline Instrumento Psicométricos & & $\mathrm{X}$ & $\mathrm{X}$ & $\mathrm{X}$ \\
\hline Modelo Matemático TRI & & & & $\mathrm{X}$ \\
\hline Banco de itens e/ou estímulos & & & & $\mathrm{X}$ \\
\hline Habilidade & & $\mathrm{X}$ & & $\mathrm{X}$ \\
\hline Comportamento & & & $\mathrm{X}$ & $\mathrm{X}$ \\
\hline
\end{tabular}

Tabela 5 - Tabela comparativa dos trabalhos relacionados

(3) testes em jogos massivos, computando as horas de utilização em relação ao seu nível de sociabilidade, procurando verificar através de um questionário psicométrico, que mede a regulação do comportamento SSQR (Short Self-Regulation Questionnaire), o nível de sociabilidade e/ou detectar o nível de dependência pela vida virtual que o usuário apresenta [11]. As principais diferenças encontradas entre os trabalhos relacionados e o PPG, são: (1) os testes com os pilotos provam a viabilidade entre testes via papel e o computadorizado, o PPG utiliza qualquer tipo de teste para aferir um perfil de habilidade e ou comportamento; (2) os testes com idosos apuram a habilidade entre diversos tipos de equipamentos e a saúde mental dos mesmos, o PPG fornece um banco de itens que prospecta, após a aferição do especialista, o perfil dos usuários de forma autônoma, ou seja, sem a necessidade de intervenção do especialista; (3) os testes com jogos massivos tentam aferir a sociabilidade dos jogadores, procurando relacionar as horas gastas no jogo e o seu nível de autoregulação, o PPG utiliza o modelo matemático TRI proposto pela Psicometria para prospectar o perfil dos avaliados oferecendo interface flexível para criação de sistemas capazes de utilizarem o PPG.

Com base nos três estudos apresentados, um comparativo se faz necessário para melhor identificar os pontos que este trabalho tem como foco. Alguns critérios foram definidos para a comparação e são explicados a seguir: a) Perfil de Usuários: identificar a geração de perfil de usuários; b) Inferência Estatística: esse critério avalia a utilização, ou não, da inferência estatística nos trabalhos relacionados; c) Instrumentos Psicométricos: avalia a existência da utilização de alguma técnica Psicométrica; d) Modelo Matemático TRI: verifica-se a utilização do modelo matemático TRI; e)

Banco de Itens e/ou Estímulos: verifica-se a utilização de bancos de itens e/ou estímulos para apli- car as técnicas de Psicometria; f) Habilidade: esse critério analisa a utilização de habilidades educacionais na composição do perfil do usuário; g) Comportamento: analisa-se a utilização de comportamentos psicológicos através de estímulos na construção do perfil dos avaliados.

\section{Considerações Finais}

As principais conclusões alcançadas no estudo foram: (1) os estudos direcionados para prospecção de perfis (habilidades e/ou comportamentos) nas áreas de educação e jogos de computadores ainda são bastante recentes; (2) por sua vez, a integração dos temas (psicometria, psicologia e computação aplicada) voltados à criação de perfis comportamentais ainda foram pouco pesquisados, mas surgem como um tema inovador e com grande potencial para exploração nos próximos anos.

Considerando o cenário exposto neste trabalho, algumas reflexões são importantes. (1) ao devolver o nível de habilidade o PPG consegue fornecer não somente um número, mas também uma analise profunda sobre cada item; (2) a tomada de decisão, ou seja, as questões de adaptação ficam por conta dos sistemas externos.

O principal trabalho futuro abrange a confecção de um sistema de prova on-line, onde o especialista criará suas questões, formulando itens a serem mensurados. $\mathrm{O}$ sistema de prova on-line interagirá diretamente com o PPG.

\section{Referências}

[1] ANASTASI, Anne. Testagem psicológica. Porto 
Alegre: Artmed, 2000.

[2] ANDRICH, David. Rasch models for measurement. Newbury Park: SAGE, 1988.

[3] BAKER, Frank B. The basics of item response theory. University of Wisconsin, 2001.

[4] FREDERICO. Measuring recognition performance using computer-based and paper-based methods. Behavior research methods: instruments, and computers, v. 23 , n. 3 , p. 341-347, 2001.

[5] MUNI, Mahmud, A mixed method for evaluating input devices with older persons, Proceedings of the 8th international ACM SIGACCESS conference on Computers and accessibility, October 23-25, 2006, Portland, Oregon, USA.

[6] PASQUALI, Luiz. Teoria da Resposta ao Item IRT: uma introdução. In.: . (Org.). Teoria e métodos de medida em ciências do comportamento. Brasília: INEP, 1996. P. 173-195.

[7] PASQUALI, Luiz. Psicometria: teoria dos testes na psicologia e na educação. Rio de Janeiro: Vozes, 2003.

[8] PASQUALI, Luiz. Fundamentos da teoria da resposta ao item - TRI: avaliação psicológica. 2003.

[9] PASQUALI, Luiz. Instrumentos psicológicos: manual prático de elaboração. Brasília, DF: LabPAM/IBAP, 1999.

[10] SANTOS, Fabrícia Damando; GUEDES, Leonardo Guerra de Rezende. Testes adaptativos informatizados baseados em teorias de resposta ao item utilizados em ambientes virtuais de aprendizagem. Porto Alegre: CINTED-UFRGS, 2005.

[11] SEAY, A. Fleming; KRAUT, Robert E.: Project massive: self-regulation and problematic use of online gaming. In: Proceedings of ACM CHI 2007: Conference on Human Factors in Computing Systems 2007. P. 829-838. 